\title{
O uso das taxas de crescimento por idade para identificação das principais etapas da transição demográfica no Brasil
}

\author{
Luana Junqueira Dias Myrrha* \\ Pamila Cristina Lima Siviero* \\ Simone Wajnman ${ }^{* * *}$ \\ Cássio M. Turra
}

\begin{abstract}
A análise do conjunto de taxas específicas de crescimento por idade de uma população, proposta por Horiuchi e Preston (1988), é uma maneira alternativa de investigar o processo da mudança da estrutura etária de uma população, bem como de examinar possíveis “marcas" deixadas pela história demográfica de cada coorte. O objetivo deste trabalho é aplicar tal metodologia à população brasileira, discutindo grandes mudanças demográficas a partir da função corrente de crescimento populacional do Brasil, observada nos períodos entre 1970 e 1980, 1980 e 1990, 1990 e 2000, 2000 e 2010. A partir dos padrões da função de taxas específicas de crescimento, simulados por Horiuchi e Preston (1988) para descrever a estrutura das taxas específicas de crescimento para cada regime de mudança demográfica, foram avaliadas possíveis marcas da história demográfica presentes nas atuais coortes brasileiras. Os resultados demonstram que as informações contidas nas taxas específicas de crescimento são bastante elucidativas com relação aos efeitos de variações nos regimes demográficos, sendo de grande utilidade em populações cujas taxas vitais não são confiáveis ou estão indisponíveis.
\end{abstract}

Palavras-chave: Taxas específicas de crescimento. Envelhecimento populacional. Mortalidade. Fecundidade.

\footnotetext{
* Universidade Federal do Rio Grande do Norte, Natal-RN, Brasil (luanamyrrha@gmail.com).

** Universidade Federal de Alfenas, Alfenas-MG, Brasil (pclsiviero@gmail.com).

${ }^{* \star \star}$ Universidade Federal de Minas Gerais, Belo Horizonte-MG, Brasil (wajnman@cedeplar.ufmg.br).

${ }^{\star \star \star \star \star}$ Universidade Federal de Minas Gerais, Belo Horizonte-MG, Brasil (turra@cedeplar.ufmg.br).
} 


\section{Introdução}

A principal característica de uma população estável é o fato de que todos os grupos de idade crescem a uma mesma taxa e a estrutura etária não se altera ao longo do tempo. Por conseguinte, a não estabilidade de uma população pode ser prontamente identificada por meio de taxas de crescimento distintas entre os grupos etários. Mais interessante é que a função de taxas específicas de crescimento por idade observada pode ser utilizada para indicar os processos que causaram as mudanças na dinâmica demográfica na história recente.

De fato, o conjunto de taxas específicas de crescimento populacional por grupos de idade, calculadas para um intervalo de tempo curto - o intervalo intercensitário, por exemplo -, carrega todas as informações sobre as mudanças demográficas ocorridas desde o nascimento da mais velha das coortes que estão sendo observadas (o grupo de idade mais elevada) até o presente. Quanto mais elevado é o grupo de idade para o qual se calcula a taxa de crescimento, maior é a carga de informações históricas contida nessa medida. Por conterem toda essa riqueza de informações e por serem, ao mesmo tempo, facilmente observáveis, não é de se estranhar que as taxas específicas de crescimento populacional por idade já tenham sido exploradas para uma série de propósitos.

Bennett e Horiuchi (1981) e Preston e Coale (1982), ao demonstrarem que as mesmas relações que conectam as funções demográficas na teoria das populações estáveis podem ser generalizadas para qualquer conjunto de taxas específicas de crescimento por idade, abriram caminho para várias aplicações. A mais direta delas é a identificação de padrões históricos de mudanças no comportamento da fecundidade, mortalidade e migrações, como em Horiuchi e Preston (1988). Outras aplicações incluem os chamados métodos da variável-r, que permitem a estimação de medidas de coorte a partir de informações demográficas obtidas em um intervalo de tempo no qual a população não é estacionária. Entre os exemplos de utilização desses métodos, destaca-se o cálculo de funções de sobrevivência a partir de distribuições de decrementos de período ajustadas segundo as taxas específicas de crescimento (MERLI, 1988; PRESTON, 1987), além da estimação do tamanho de coortes não extintas a partir dos óbitos de coortes adjacentes e do crescimento demográfico por idade (COALE; CASELLI, 1990). Nessa mesma linha metodológica, Preston e Coale (1982) mostraram que, apenas com as taxas específicas de crescimento e a distribuição observada dos nascimentos segundo idade das mães, é possível estimar com precisão a taxa líquida de reprodução de uma população. A estratégia desenvolvida pelos autores foi aplicada por Cai (2008) para certificar o nível de fecundidade reportado nas estatísticas chinesas do início deste século. Com base no mesmo método, Preston e Wang (2007) mediram o efeito da migração internacional nas taxas de crescimento de longo prazo de vários países e Javique et al. (2013) estimaram as consequências da migração interna e internacional para a dinâmica populacional das províncias de Cuba.

Neste trabalho, seguindo os padrões simulados por Horiuchi e Preston (1988), são analisadas as informações contidas nas taxas de crescimento por grupos de idade 
quinquenais, obtidas comparando-se estimativas populacionais para 1970, 1980, 1990, 2000 e 2010, como forma de identificar as principais mudanças históricas que ocorreram nas variáveis demográficas no Brasil. Nas próximas seções, primeiramente, são revisadas as principais etapas do processo de transição demográfica brasileira. Em seguida, descrevem-se o método e os dados utilizados e são analisados os resultados extraídos pelo uso das taxas específicas de crescimento. Por fim, discute-se como esse tipo de análise pode ser útil não apenas para inferir sobre os processos passados da dinâmica populacional, mas também para a previsão de oscilações futuras nos tamanhos dos subgrupos populacionais, o que pode ser de grande utilidade para o planejamento das demandas de subgrupos específicos. Também é indicada a conveniência da comparação dos perfis etários de taxas de crescimento ao longo do tempo como um instrumento valioso na verificação da qualidade dos dados censitários.

\section{Antecedentes}

As mudanças ao longo das distintas fases da transição demográfica brasileira já foram bem documentadas na ampla literatura que estabeleceu alguns fatos básicos, que são descritos a seguir, de forma bastante sucinta. No período de 1940 a 1970, a redução do nível da mortalidade teve como consequência o rápido declínio da taxa bruta de mortalidade (TBM), em relação à taxa bruta de natalidade (TBN), o que acelerou o crescimento populacional brasileiro. A partir da década de 1970, a taxa de crescimento populacional continuou a aumentar, embora em ritmo decrescente, devido à redução rápida e generalizada do nível da fecundidade. Entretanto, neste período, a TBN não diminuiu o seu valor na mesma proporção do declínio do nível da fecundidade, uma vez que um número crescente de mulheres de coortes anteriores à queda da fecundidade ainda chegava à idade reprodutiva. Consequentemente, as taxas de crescimento correntes não sofreram por completo o impacto do decréscimo da fecundidade (MOREIRA; CARVALHO, 1992). Esse fenômeno, conhecido como inércia populacional, juntamente com a redução da TBM, manteve a taxa de crescimento ainda bastante elevada: enquanto a taxa de fecundidade total (TFT) caiu 25,9\%, a taxa de crescimento reduziu-se em apenas 14\%. Um declínio mais significativo na taxa de crescimento, de 21\%, foi observado somente entre 1980 e 1991 (CARVALHO, 2004). As estimativas de Wong e Carvalho (2006), com base no Censo Demográfico de 2000, apontam para a continuidade do declínio sustentado da fecundidade e sugerem que a mortalidade continuará caindo em todas as idades, havendo, porém, uma maior redução nas idades mais avançadas. Embora ambas as tendências acelerem o processo de mudanças na estrutura etária brasileira, envelhecendo a população, o declínio da fecundidade é considerado o principal responsável pelas transformações observadas, seguido pelo declínio da mortalidade (MOREIRA, 1997; CARVALHO; GARCIA, 2003; CARVALHO, 2004; CAMARANO, 2002; DIAS JÚNIOR; COSTA, 2006; WONG; CARVALHO, 2006; CARVALHO; WONG, 2008; MYRRHA, 2009).

Em geral, atribui-se um papel muito pequeno, praticamente nulo, à migração na transformação recente da estrutura etária da população brasileira. Da segunda metade do 
século XIX até os anos 1930, período que antecedeu as grandes mudanças na fecundidade e mortalidade, o Brasil apresentou saldo migratório - SM (diferença entre o número de imigrantes e o de emigrantes) - positivo, em função do alto fluxo de imigrantes. Mas os fluxos migratórios reduziram de intensidade entre as décadas de 1930 e 1980, com exceção dos anos que sucederam a Segunda Grande Guerra, fazendo com que a população brasileira passasse a ser considerada fechada (BELTRÃO; CAMARANO, 1997). Entre 1980 e 1990, uma mudança no padrão de migração fez com o que o Brasil experimentasse uma perda líquida de aproximadamente 1,6\% da população com mais de dez anos de idade (CARVALHO, 1996). Nos anos 1990, o SM internacional do Brasil foi consideravelmente menor do que na década anterior (apenas 0,4\% da população total), mas permaneceu negativo, uma vez que o número de emigrantes brasileiros continuou mais elevado do que o de imigrantes (CARVALHO; CAMPOS, 2006).

Poucos são os estudos que, de fato, mensuraram sistematicamente a contribuição dos componentes da dinâmica demográfica sobre o processo de mudança da estrutura etária brasileira. Moreira (1997) projetou a população total do país, entre 1995 e 2020, segundo diferentes cenários para os componentes demográficos, de forma a isolar o efeito de cada uma delas sobre as mudanças na estrutura etária. Os resultados do estudo demonstraram que a fecundidade é o componente definidor da variação da estrutura etária brasileira neste período. Fígoli e Wong (2003) mediram o momentum populacional em cinco países da América Latina, incluindo o Brasil, segundo diferentes métodos. O exercício mostrou que o grau de envelhecimento da estrutura etária depende de variações na fecundidade, de diferenças na história demográfica de cada coorte e também, em grande medida, do nível inicial de mortalidade e das suposições com relação ao seu comportamento no futuro. Mais recentemente, Myrrha (2009) mensurou a variação da idade média da população brasileira segundo diferentes métodos de decomposição, para avaliar o papel das mudanças nas taxas de fecundidade e mortalidade. O trabalho da autora confirmou o papel preponderante das mudanças da fecundidade para o envelhecimento populacional, mas também sugeriu um papel crescente da mortalidade nas décadas que estão por vir.

Em outro estudo amplamente citado na literatura, Bercovich e Madeira (2004) examinaram as diferenças nos tamanhos de coortes sucessivas no Brasil, entre 1910 e 2000, com o objetivo de identificar possíveis descontinuidades demográficas, especialmente aquelas expressas por meio de mudanças no número de nascimentos. Além disso, as autoras investigaram variações no tamanho absoluto e relativo da população de jovens adultos em áreas geográficas específicas. Os resultados encontrados serviram como referência para aplicações em diferentes áreas do conhecimento (ver, por exemplo, MUNIZ, 2002).

Ao contrário dos estudos anteriores, neste artigo não são medidas as variações absolutas no tamanho de coortes sucessivas. Seguindo os passos indicados por Horiuchi e Preston (1988), descritos na seção a seguir, utilizam-se funções de taxas de crescimento por idade, observadas em períodos recentes, para detectar os principais fatos que marcaram a história demográfica da população brasileira no século XX. 


\section{Metodologia}

O método empregado neste trabalho trata de analisar as mudanças demográficas das últimas décadas no Brasil, por meio da inspeção do conjunto de taxas específicas de crescimento populacional, de forma a mapear as possíveis “marcas” deixadas pela história de cada coorte, como proposto por Horiuchi e Preston (1988). Essas informações têm sido muito pouco utilizadas, apesar de estarem amplamente disponíveis; com apenas o número de pessoas em cada idade em dois pontos no tempo, as taxas de crescimento podem ser diretamente calculadas.

O número de pessoas por grupo etário e período utilizado para o cálculo das taxas específicas de crescimento - daqui em diante designadas TECs - é proveniente de estimativas da população brasileira fornecidas pelo Centro Latino Americano e Caribenho de Demografia das Nações Unidas (Celade), para os anos de 1970, 1980, 1990, 2000 e 2010. As estimativas têm como base as informações dos Censos Demográficos realizados pelo Instituto Brasileiro de Geografia e Estatística (IBGE). Os dados censitários originais foram ajustados pelos pesquisadores do Celade, com o objetivo de evitar omissões e inconsistências temporais (CELADE, 2011). As TECs foram calculadas com base na equação de crescimento exponencial que assume uma taxa de crescimento populacional constante no intervalo de tempo em estudo (PRESTON et al., 2001):

$$
r(a, t / t+n)=\frac{\ln \left[\frac{N_{a}(t+n)}{N_{a}(t)}\right]}{n}
$$

onde: $r(a, t)$ é taxa de crescimento do grupo etário $a ; N_{a}(t)$ corresponde à população do grupo etário a no período $(t) ; N_{a}(t+n)$ é a população do grupo etário a no período $(t+n)$; e $n$ refere-se ao período intercensitário.

Horiuchi e Preston (1988) apresentam alguns conjuntos hipotéticos de funções de taxas específicas de crescimento que correspondem a cenários estilizados de mudanças demográficas, descrevendo, assim, um padrão típico de curvas de taxas específicas de crescimento para cada tipo de mudança demográfica isoladamente. Tendo como ponto de partida uma população estável hipotética, os autores simularam três cenários alternativos de declínio da mortalidade (moderado, rápido e declínio interrompido 30 anos após seu início), mantendo a fecundidade constante, além de um cenário com declínio da fecundidade, mantendo mortalidade constante. São reproduzidos aqui os resultados do primeiro cenário de queda da mortalidade (declínio moderado) e o de queda da fecundidade, uma vez que eles representam referências claras para a interpretação dos perfis de taxas específicas de crescimento, observados para a população brasileira. ${ }^{1}$

\footnotetext{
${ }^{1}$ Os detalhes de cada cenário, bem como os padrões gráficos para os cenários de queda rápida e queda interrompida da mortaldade, podem ser vistos em Horiuchi e Preston (1988, p. 433-35).
} 
Com uma tabela de vida do Modelo Oeste, expectativa de vida de 40 anos, fecundidade constante e população fechada a migrações, no primeiro cenário, simulou-se o efeito puro de um declínio da mortalidade contínuo, a um ritmo de 0,5 ano de ganho na esperança de vida ao nascer por ano-calendário. Neste cenário de redução moderada da mortalidade, as curvas de taxas específicas de crescimento foram observadas em quatro intervalos de períodos: 10 a 15 anos, 25 a 30 anos, 45 a 50 anos e 70 a 75 anos após o início da mudança. 0 Gráfico 1 apresenta os resultados para cada período de observação. Ao longo do tempo, as taxas específicas de crescimento aumentam para todas as idades, havendo, no entanto, uma mudança significativa no perfil da curva. No período de 10 a 15 anos após o início do declínio, identifica-se um perfil em forma de U. Seguindo a estrutura típica da função de mortalidade por idade e considerando um nível inicial de mortalidade bastante elevada, este padrão corresponde ao fato de que as crianças que nascem ou que ainda estão na primeira infância logo após o início de queda da mortalidade tendem a ser fortemente afetadas. Os adultos também são crescentemente afetados, à medida que a idade aumenta. Os menos beneficiados pelo declínio são as crianças que tinham entre 5 e 9 anos no início da mudança. Com a extensão dos ganhos de longevidade no primeiro ano de vida para todas as novas coortes e o envelhecimento das primeiras coortes beneficiadas, o perfil vai se tornando mais horizontal. Vale notar que a taxa de crescimento permanece elevada para a coorte que primeiro se beneficiou da redução da mortalidade infantil, relativamente às coortes mais jovens, uma vez que, com a continuidade do processo de declínio no nível de mortalidade geral, o decréscimo nas taxas específicas de mortalidade nas idades mais jovens torna-se relativamente menor. Além disso, é entre os mais velhos que vão se concentrar as maiores taxas de crescimento, em função de o padrão de ganhos de longevidade tornar-se cada vez mais restrito às idades avançadas, levando ao envelhecimento populacional.

No cenário de queda da fecundidade (Gráfico 2) mantendo a mortalidade constante, os efeitos sobre a função de taxas específicas de crescimento são mais diretos. No primeiro ano em que a fecundidade começa a declinar, a partir de uma população previamente estável, a coorte com menos de um ano exibe uma taxa de crescimento menor do que das coortes mais velhas. Após 15 anos de queda da fecundidade, toda a população menor de 15 anos está registrando taxas de crescimento menores do que no cenário de estabilidade. Além disso, a partir desse ponto, a coorte que experimentou taxas menores de crescimento em função dos declínios na fecundidade entra em idade reprodutiva, o que diminui ainda mais a taxa de crescimento das coortes que estão nascendo. Nos primeiros anos de queda, a população em idade reprodutiva está crescendo a uma taxa que, aproximadamente, compensa o declínio anual das taxas específicas de fecundidade, produzindo um breve período de crescimento zero nos nascimentos. ${ }^{2}$ Como mostram as curvas referentes aos períodos de tempo mais distantes (25-30 anos ou mais), quando as coortes nascidas do crescimento zero entram

\footnotetext{
$\overline{2}$ Nessa simulação, a população cresce a uma taxa de 1,54\% ao ano e o declínio imposto às taxas de fecundidade é de $1,5 \%$ ao ano.
} 
na idade reprodutiva, o número absoluto de nascimentos começa a diminuir e as taxas específicas de crescimento se tornam negativas.

\section{GRÁFICO 1}

Simulação das taxas específicas de crescimento por idade desde o início do declínio da mortalidade, em um cenário de queda moderada da mortalidade

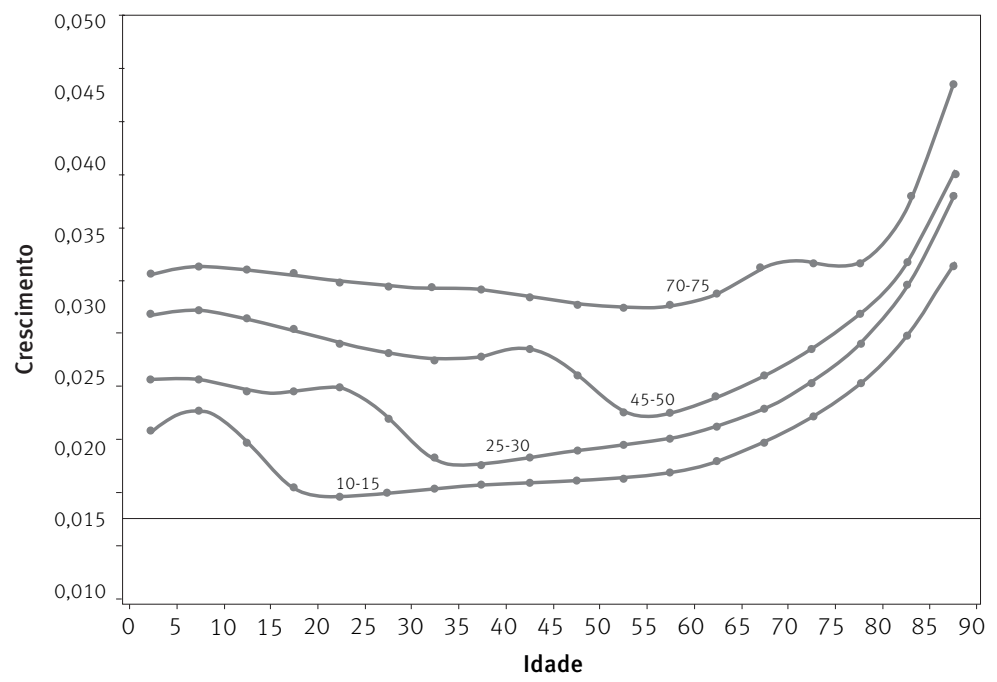

Fonte: Extraído de Horiuchi e Preston (1988)

Nota: Reproduzido com autorização.

\section{GRÁFICO 2}

Simulação das taxas específicas de crescimento por idade desde o início do declínio da fecundidade, no cenário de queda exclusiva da fecundidade

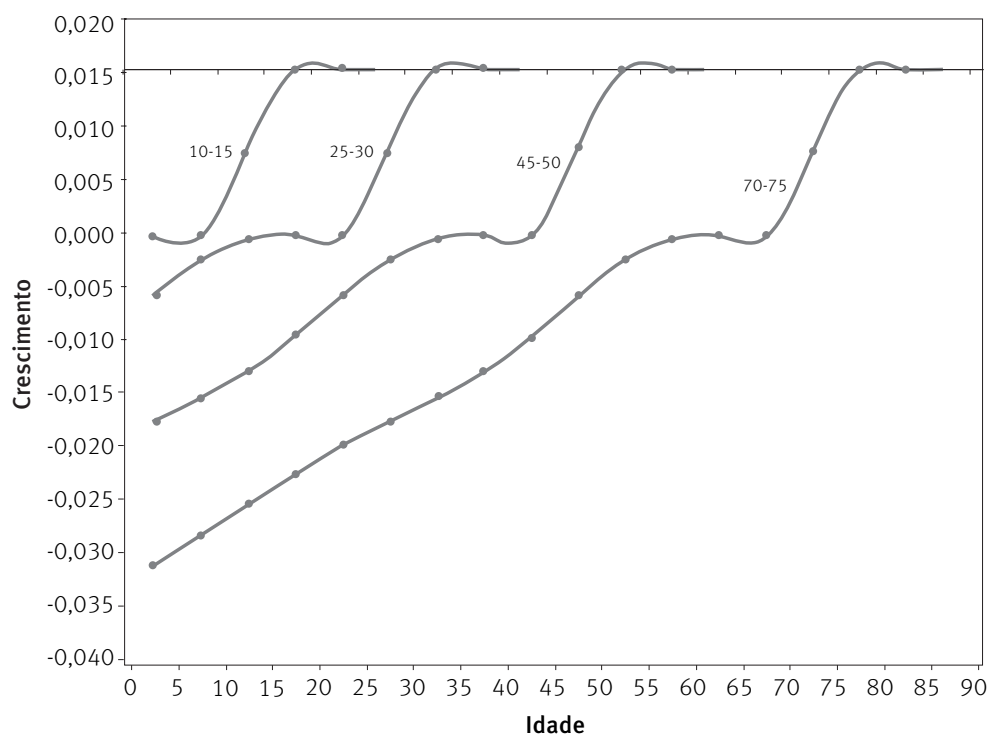

Fonte: Extraído de Horiuchi e Preston (1988).

Nota: Reproduzido com autorização. 


\section{Resultados}

O Gráfico 3 apresenta a função de taxas específicas de crescimento por idade para o período entre 1970 e 1980. Com o auxílio de um diagrama de Lexis (Figura 1), foram estabelecidas associações entre o padrão da curva de taxas de crescimento do Gráfico 3 e as mudanças demográficas subjacentes, identificando as coortes que vivenciaram as principais mudanças, bem como o provável momento de sua ocorrência. No diagrama estão representadas as coortes cujos comportamentos das TECs deseja-se aqui chamar a atenção. São quatro as evidências mais relevantes enumeradas no diagrama e descritas a seguir.

1. Conforme discutido no Gráfico 1, mudanças nas taxas específicas de mortalidade tendem a afetar mais fortemente os grupos de idade mais jovens e os mais velhos, com o que se espera encontrar, nos estágios iniciais de queda da mortalidade, um padrão em forma de U para as taxas específicas de crescimento (HORIUCHI; PRESTON, 1988, p. 433), que indicaria as coortes mais beneficiadas pelo processo. Com efeito, pode-se identificar esse formato de curva em U no Gráfico 3, do grupo etário de 25-29 anos até as idades mais velhas. Além disso, o pico da TEC no grupo de 25-29 anos, no Gráfico 3, pode ser associado à acentuada queda da mortalidade infantil ocorrida entre as décadas de 1940 e 1950 (pós-guerra). ${ }^{3}$

2. No Gráfico 3, o ponto mais baixo da curva em forma de U, originada pela queda da mortalidade, corresponde ao grupo etário de 40-44 anos. Essa seria, então, a coorte que menos se beneficiou da redução da mortalidade, uma vez que, quando o processo começou, as pessoas dessa coorte já tinham vencido a primeira infância e não eram ainda suficientemente adultas para se beneficiar dos ganhos em longevidade. Dito de outra forma, esta coorte estava no ponto mais baixo (e menos sensível) da curva de mortalidade por idade. É com base nessa lógica que Horiuchi e Preston (1988) propõem que a duração do declínio da mortalidade pode ser identificada pela subtração de 7,5 anos (ponto médio do grupo etário de menor nível de mortalidade) da idade que representa o ponto mínimo da curva das TECs. De acordo com essa proposição, a duração da queda da mortalidade pode ser calculada da seguinte forma: 42,5 (ponto médio do grupo etário 40 a 44 anos) - 7,5 = 35 anos. Assim, considerando 1975 o ponto médio do período analisado, a queda da mortalidade teria se intensificado a partir de 1940 (ou seja, 35 anos antes de 1975), reiterando os achados da literatura sobre o início da transição da mortalidade no Brasil (IBGE, 1999). No Gráfico 4, que apresenta as curvas referentes aos períodos 1980-1990,1990-2000 e 2000-2010, observa-se que essa curva em “U” se desloca para os grupos etários subsequentes, mantendo o mesmo padrão de comportamento descrito até aqui. Isso corrobora a noção de que as marcas de fenômenos demográficos que afetam a história de uma coorte ficam impressas por longos períodos, podendo ser utilizadas como fonte de informação histórica, muitas décadas depois.

\footnotetext{
${ }^{3} 0$ exame da série de taxas de mortalidade infantil para as décadas de 1930 a 1960 no Brasil (IBGE, 1999) indica que a maior redução ocorreu entre meados dos anos 1940 e 1950, exatamente o período de nascimento das coortes com 25 a 29 anos em 1970 e 1980.
} 
GRÁFICO 3

Taxas específicas de crescimento, por grupos de idade Brasil - 1970-1980

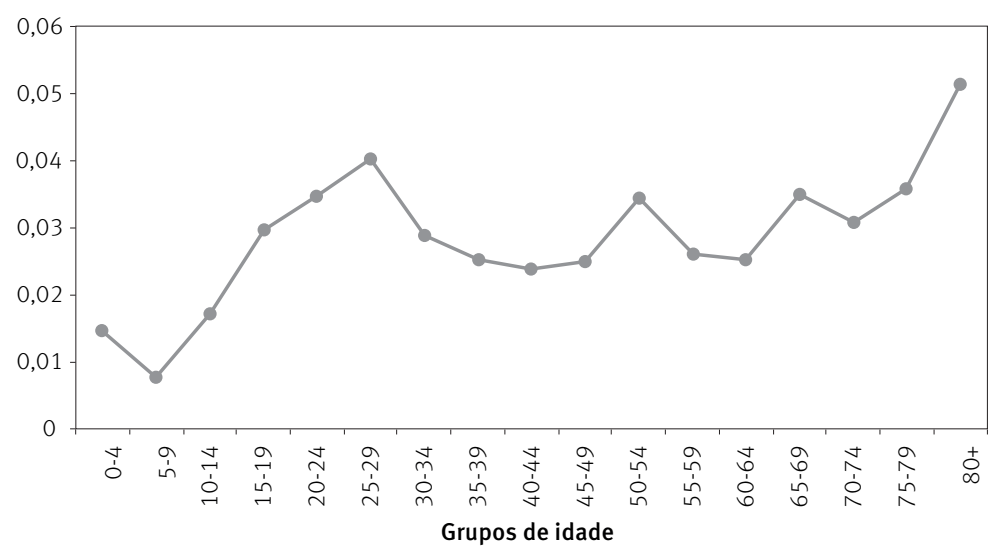

Fonte: IBGE. Censos Demográficos. Estimativas do Celade.

FIGURA 1

Diagrama de Lexis: representação das taxas de crescimento por idade para períodos e coortes selecionados no Brasil

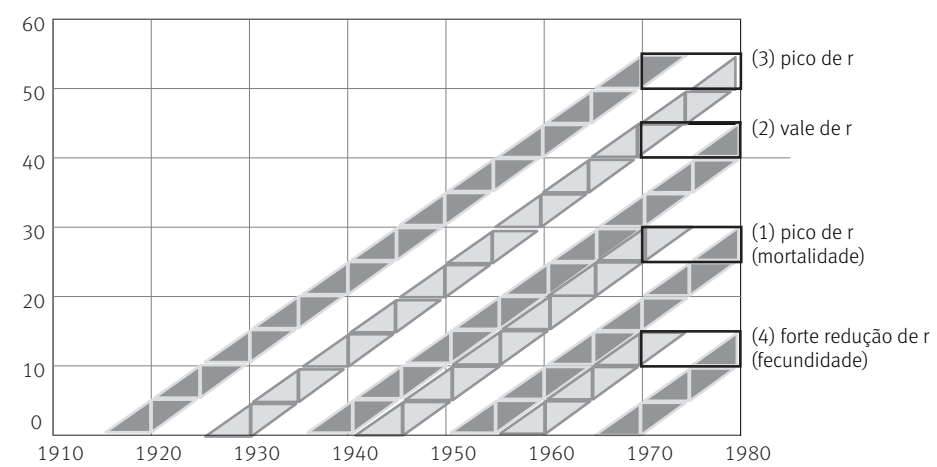

Nota: Para efeito de ilustração, essa figura foi construída considerando que os dados referem-se a 1ํ de janeiro do ano censitário.

GRÁFICO 4

Taxas específicas de crescimento, por grupos de idade (estimativas do Celade) Brasil - 1980-2010

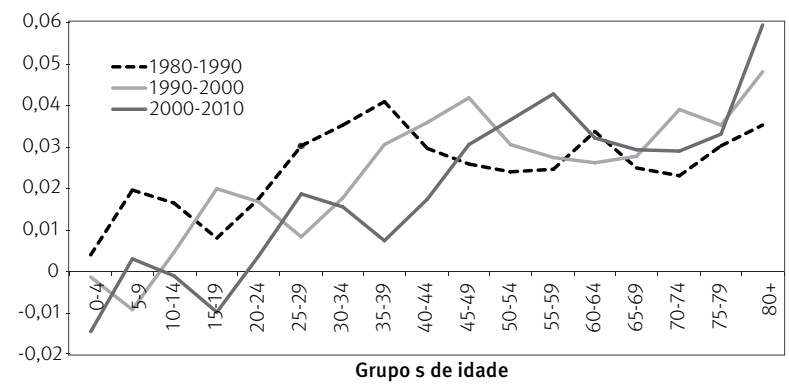

Fonte: IBGE. Censos Demográficos. Estimativas do Celade. 
3. Voltando ao Gráfico 3, observa-se haver um segundo pico de TEC para o grupo etário de 50-54 anos no período 1970-80, o qual corresponde a um expressivo crescimento do tamanho da coorte nascida em 1925-30 em relação àquela nascida em 1915-20. As mudanças demográficas responsáveis por tal comportamento devem ser objeto de pesquisa mais detalhada, já que correspondem a coortes nascidas em um período da história menos documentado da população brasileira. De qualquer forma, é possível que este crescimento reflita, em menor escala, alguma queda da mortalidade ao longo de vida dessa coorte - especialmente durante o período de maternidade - e, em maior escala, o afluxo de imigrantes adultos jovens ao Brasil no pós-Segunda Guerra. Além disso, vale notar que a elevada TEC do grupo de 25-29 anos, descrita no item anterior, pode estar indiretamente relacionada à alta taxa de crescimento do grupo de 50-54 anos. Assumindo-se uma idade média à maternidade de 25 anos, parte do crescimento da coorte de 25-29 anos, em 1970-1980, seria efeito indireto do aumento no número de progenitores representados pelo grupo de 50 a 54 anos.

4. A faixa etária de 10-14 anos apresenta uma acentuação no padrão de redução das TECS desde o grupo de 25-29 anos. Essa mudança corresponde à queda da natalidade, em função da transição de fecundidade, que começa a reduzir o crescimento das coortes de nascidos entre 1955-60 e 1965-70. Mais acentuado, ainda, é o declínio verificado entre as coortes nascidas nos períodos 1960-65 e 1970-75 (taxa de crescimento do grupo 5 a 9 anos no Gráfico 3). Uma taxa de crescimento mais elevada para o grupo etário 0-4 anos sugere, em primeiro lugar, o arrefecimento da queda da natalidade no final dos anos 1970, já que é nesse período que nasceram os filhos das gerações cujo crescimento populacional mais do que compensou a queda no número de filhos tidos por mulher. Este efeito estaria retratado na correspondência entre as elevadas TECs para os grupos de 25 a 29 e 0 a 4 anos, aproximadamente gerações de mães e filhos. Além disso, a maior TEC para o grupo de 0 a 4 anos também é consistente com a aceleração na queda da mortalidade infantil a partir dos anos 1970, depois de um período de estagnação durante a década de 1960, conforme relatos na literatura (IBGE, 1999).

O Gráfico 4 traz evidências adicionais quanto ao processo de queda da fecundidade. A combinação entre a diminuição do número de filhos tidos por mulher e a redução da população em idade reprodutiva faz com que a taxa de crescimento dos grupos com menos de dez anos de idade sejam negativas pela primeira vez no período 1990-2000. Em outras palavras, uma TEC negativa para o grupo 5-9 anos na curva de 1990-2000 indica que foi entre 1990 e 1995 que se deu, pela primeira vez no país, uma queda no número absoluto de nascimentos, certamente porque foi nesse período que chegaram à idade reprodutiva as mulheres nascidas sob o regime de fecundidade declinante. Na curva referente às TECs de 2000-2010, esta taxa negativa reaparece para o grupo etário 15-19 anos, confirmando o mesmo, ou seja, entre 15 a 19 anos antes de 2010, ou seja, entre 1990 e 1995, o número de nascimentos começou a decrescer no Brasil.

Adicionalmente, as TECs do Gráfico 4 mostram também que os chamados efeitos de "ecos demográficos" causam oscilações no número de nascimentos e acabam por gravar suas marcas por toda a pirâmide etária. Nota-se que a TEC de 1990-2000 relativa ao grupo 0-4 anos assume um valor próximo de 0 (zero), indicando que entre 1995 e 2000 houve um aumento do número de nascimentos com relação ao período anterior. Essa reversão na tendência de queda é facilmente explicada quando se observa a correspondência inequívoca 
entre os aumentos das TECs dos grupos de 0-4 e 15-19 anos de idade, ou seja, idades correspondentes aos nascimentos e à entrada das mulheres no período reprodutivo. As mesmas evidências se apresentam na curva relativa ao período 2000-2010, com as idades deslocadas em dez anos.

\section{Utilidades práticas dos perfis das TECs}

Evidentemente, as “marcas” encontradas nas curvas das TECs apenas confirmam o que se sabe por outros caminhos sobre a história demográfica recente do Brasil. No entanto, a intenção neste trabalho é elucidar como as TECs podem se constituir numa poderosa ferramenta de conhecimento da história demográfica, na ausência de outras evidências. Supondo-se, por exemplo, que não houvesse, no Brasil, censos populacionais confiáveis antes de 1990 e que pouco se soubesse sobre a dinâmica demográfica anterior, com base apenas na análise dos perfis de taxas de crescimento dos períodos 1990-2000 e 20002010 - que são inteiramente consistentes entre si -, seria possível inferir que o declínio da mortalidade teria se iniciado na década de 1940 e a queda da fecundidade, na de 1960.

Além do conhecimento retrospectivo, as TECs também auxiliam na previsão das oscilações populacionais futuras de curto prazo, já que, com base nos seus perfis, pode-se prever, com chances mínimas de erros, qual é o crescimento esperado para os subgrupos etários específicos da população. Assim, espera-se que, quando compararmos o Censo de 2020 com o de 2010, o grupo que terá maior TEC, excluindo o grupo aberto de 80 anos e mais, será o de 65-69 anos e, no Censo de 2030, o de 75-79 anos, potencializando o efeito do envelhecimento populacional causado pela queda dos nascimentos, com a chegada a estas faixas etárias da coorte mais impactada pelo início da queda da mortalidade. Sabe-se também que, entre os adultos acima de 20 anos, o grupo etário de menor crescimento no período 2010-2020 é o de 35-39 anos, precisamente o que teve menor taxa de crescimento no período 2000-2010. Previsões desse tipo são uma aplicação direta das funções de TECs ao longo do tempo e ajudam a explicar os "zigue-zagues" da pirâmide etária de uma população, muitas vezes mal compreendidos pelo analista leigo. Nesse sentido, vale ressaltar que a análise a partir das taxas de crescimento evidencia uma dimensão menos estática da distribuição etária da população, que não é automaticamente obtida a partir do exame das pirâmides etárias, qual seja, a variação do tamanho dos grupos de idade ao longo do tempo.

Finalmente, é oportuno destacar também que os perfis etários das TECs podem ser muito úteis na verificação da qualidade dos dados censitários. Como observado na seção metodológica deste trabalho, optou-se por utilizar os dados censitários produzidos pelo IBGE e ajustados pelo Celade. Se, em vez destes dados ajustados, tivessem sido usados os dados censitários coletados diretamente pelo IBGE, sem qualquer correção, seriam obtidos os resultados apresentados no Gráfico 5. Comparando-se os perfis exibidos nos Gráficos 4 e 5, é fácil perceber que as TECs obtidas diretamente dos Censos são menos consistentes e sugerem haver algum tipo de erro nos dados, já que dificilmente a dinâmica demográfica 
- ou seja, os comportamentos de fecundidade, mortalidade e migração - por si só pode explicar tais resultados.

\section{GRÁFICO 5 \\ Taxas específicas de crescimento, por grupos de idade (dados censitários) \\ Brasil - 1980-2010}

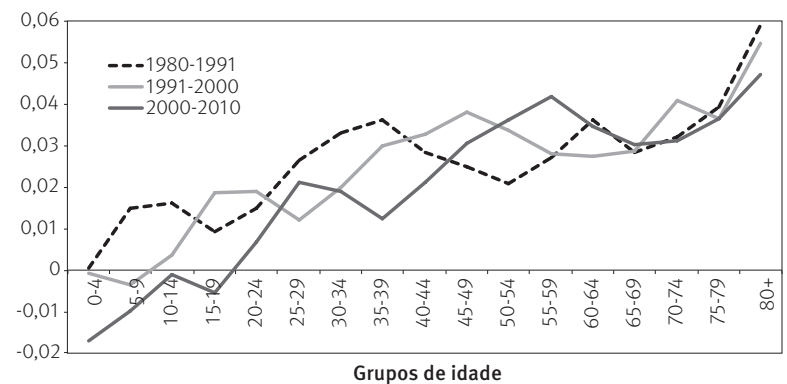

Fonte: IBGE. Censos Demográficos

Na ausência de problemas na qualidade dos dados, as TECs de censos sucessivos devem ter um comportamento previsível e bastante estável. Por exemplo, tomando-se o primeiro ponto da curva de TECs de 1980-1990, referente ao grupo de 0-4 anos, deve-se esperar que, se os dados tiverem qualidade perfeita e a população for fechada, a diferença entre esta taxa e a observada no grupo de 10-14 anos na curva referente ao período 1990-2000, bem como a verificada no grupo de 20-24 anos na curva de 2000-2010, deverá corresponder a apenas o efeito da mortalidade que atuou diferenciadamente para as duas coortes que estão sendo comparadas, a de 0-4 anos em 1980 e a de 0-4 anos em 1990. Considerando que a diferença no número de nascimentos já está captada na TEC do grupo de 0-4 anos, se houvesse rigorosamente o mesmo regime de mortalidade para ambas as coortes, deveriam ser encontrados valores idênticos para TEC do grupo 10-14 anos, no período 1990-2000, e a TEC do grupo 20-24, no período 2000-2010. Considerando, mais realisticamente, que a mortalidade continua em declínio, mas que o período de tempo transcorrido entre as experiências das duas coortes é reduzido, pode-se aceitar apenas uma TEC ligeiramente ascendente, o que é observado no Gráfico 4. No entanto, no Gráfico 5, relativo aos dados censitários sem correções, as variações nas TECs correspondentes são muito maiores. Atentando-se novamente para o grupo 0-4 anos no período 1980-1990, verifica-se que a TEC é ligeiramente negativa, tornando-se positiva no ponto correspondente na curva do período de 1990-2000, ou seja, na idade 10-14 anos. Ora, se estes valores pudessem ser explicados apenas pela dinâmica demográfica, isso indicaria que a redução no número de crianças de 0 a 4 anos, entre 1980 e 1990, teria sido compensada por uma espetacular queda na mortalidade infanto-juvenil entre coortes vizinhas (crianças de 0-4 anos em 1980 e em 1990), que tornaria a coorte mais velha maior do que a mais jovem. E procedendo com a análise para grupos etários posteriores, encontram-se novos aumentos das TECs que seriam ainda menos prováveis, nas idades adultas em que não há reduções de mortalidade que possam afetar significativamente coortes vizinhas. 
A exceção fica por conta das idades acima de 70 anos, já que as TECs desses grupos etários são mais afetadas pelo decréscimo no nível geral de mortalidade do que a dos grupos adultos mais jovens, como demonstram as simulações do Gráfico 1, realizadas por Horiuchi e Preston (1988). Ou seja, nas idades mais avançadas, espera-se, de fato, um aumento das taxas de crescimento entre coortes de mesma idade em décadas sucessivas. Embora esse padrão possa ser observado tanto para as estimativas do Celade quanto para os dados censitários, ainda assim há discrepâncias entre as duas fontes. A mais importante delas é a redução na taxa de crescimento do grupo aberto de 80 anos e mais nos sucessivos períodos intercensitários, padrão que não se observa nas estimativas produzidas pelo Celade: a TEC diminui de $5,9 \%$ para $4,7 \%$ quando calculada com os dados censitários, ao passo que ela aumenta de 3,5\% para 5,9\% pelas estimativas do Celade. Essa diferença parece ser explicada por taxas intercensitárias que são especialmente mais altas no período 1991-1980 nos dados censitários.

A comparação das TECs nas duas fontes de dados fica ainda mais evidente no Gráfico 6. Em cada ponto no eixo das abscissas, estão representadas as taxas de crescimento entre coortes de mesma idade em décadas sucessivas. Os dois painéis ilustram com clareza a maior variabilidade das TECs, ao longo das décadas, quando utilizados os dados censitários; resultado que corrobora o que havia sido analisado anteriormente. O ponto 11, por exemplo, corresponde à taxa de crescimento entre as coortes que têm idade 5054 anos em 1980 e 1990, 60-64 anos em 1990 e 2000 e 70-74 anos em 2000 e 2010. Nesse caso, a TEC aumenta de 0,024 para 0,029 nos dados do Celade, comparado a uma elevação significativamente maior, de 0,021 para 0,031, nos dados censitários. Mais uma vez, a exceção fica por conta das idades mais velhas, em que era esperado exatamente o efeito inverso, isto é, uma maior variação do que a apresentada pelos dados censitários.

\section{GRÁFICO 6 \\ Taxas de crescimento entre coortes de mesma idade em décadas sucessivas \\ Brasil - 1980-2010}
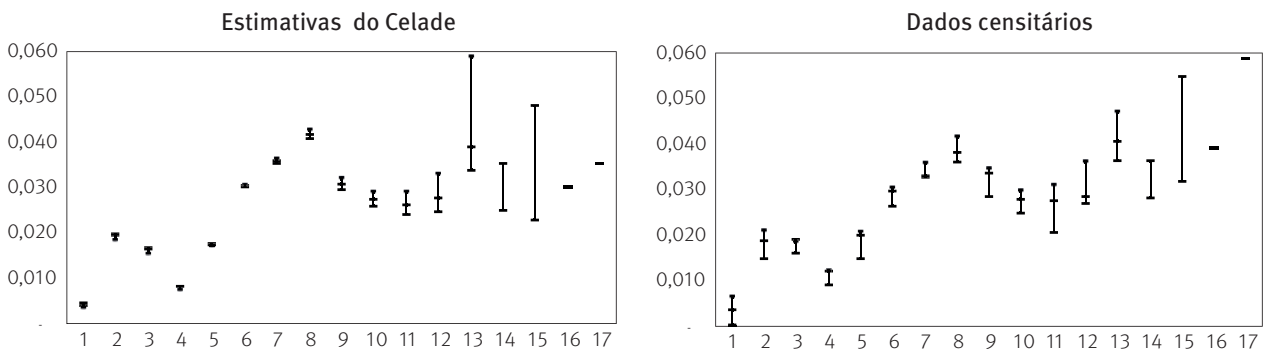

Fonte. IBGE. Censos Demográficos. Estimativas do Celade.

Nota: Cada ponto no eixo x compara a taxa de crescimento entre duas coortes de mesma idade em décadas sucessivas. Por exemplo, 0 ponto 1 indica a taxa de crescimento entre as coortes que têm idade 0-4 em 1980 e 1990, 10-14 em 1990 e 2000 e $20-24$ em 2000 e 2010. 0 ponto 2 indica a taxa de crescimento entre as coortes que têm idade 5-9 em 1980 e 1990, 15-19 em 1990 e 2000 e 25-29 em 2000 e 2010; e assim, sucessivamente. 


\section{Considerações finais}

A estrutura etária de uma população carrega, por muitas décadas, as marcas da dinâmica demográfica passada. Em geral, os estudos limitam-se a analisar a distribuição de pessoas por idade, em diferentes pontos no tempo. Uma alternativa metodológica complementar para desvendar a dinâmica demográfica passada é examinar funções de taxas de crescimento populacional específicas por idade em determinado período de tempo, como sugerem Horiuchi e Preston (1988). A principal vantagem desse tipo de análise é sua simplicidade e a facilidade de se obter um grande conteúdo de informações históricas, a partir de taxas de crescimento calculadas com base apenas em dois censos recentes e do conhecimento prévio dos padrões típicos de efeito das mudanças demográficas sobre essas taxas.

Neste trabalho, analisaram-se as funções de taxas de crescimento por idade da população brasileira nos períodos de 1970 a 1980, 1980 a 1990, 1990 a 2000 e 2000 a 2010, identificando a fase de início da queda da mortalidade, nos anos 1940, o período do início de redução da natalidade, na década de 1960, e o provável efeito do afluxo de imigrantes internacionais jovens adultos, no segundo quinquênio dos anos 1940 (pós-guerra).

Vale notar que o exame dos perfis das TECs auxilia diretamente no entendimento de como as variáveis demográficas concorrem para o envelhecimento da estrutura etária. Verifica-se que o declínio da mortalidade produz efeitos em ambas as direções: por um lado, gera taxas específicas de crescimento mais elevadas para as idades mais velhas relativamente às mais jovens, contribuindo assim para o envelhecimento da população; por outro lado, causa forte descontinuidade positiva no crescimento das coortes nascidas logo após o início do declínio, rejuvenescendo a população, embora esse efeito tenda a ser neutralizado à medida que o tempo passa e essa coorte envelhece. Quanto ao efeito da queda da fecundidade, verificou-se que, uma vez iniciado o seu processo, as taxas de crescimento dos grupos etários mais jovens declinam rapidamente e o peso desse componente passa a ser muito mais determinante para o envelhecimento do que o da queda da mortalidade, com os grupos mais jovens experimentando taxas de crescimento cada vez mais negativas.

Toda essa análise sugere que as taxas específicas de crescimento, facilmente extraídas das informações censitárias atuais, são bastante informativas com relação à história demográfica das coortes e devem ser mais bem exploradas, podendo ser de grande utilidade quando as tendências dos eventos demográficos históricos não são conhecidas ou as informações não são confiáveis.

As taxas de crescimento também são úteis ao indicarem o provável ritmo de crescimento de grupos de idade específicos, no futuro. Além disso, sua análise sugere possíveis inconsistências nos dados populacionais por idade, ao longo do tempo. Não se pretende, aqui, discutir a validade e adequação dos ajustes realizados pela equipe técnica do Celade em suas estimativas. A intenção desse estudo foi apenas apontar que a análise da variável $r$ presta-se, também, para elucidar possíveis problemas de qualidade dos dados censitários brutos. Neste contexto, uma questão a ser mais bem examinada é a mortalidade de idosos. 
Os deslocamentos das curvas de TECs para esses grupos indicam ganhos expressivos nos períodos mais recentes, mas seria necessário averiguar o quanto dessa variação devese à qualidade dos dados e quanto, de fato, espelha a verdadeira variação nas taxas de mortalidade em idades avançadas.

\section{Referências}

BELTRÃO, K. I.; CAMARANO, A. A. Cálculo de saldos e taxas líquidas de migração internacional. In: ENCONTRO NACIONAL SOBRE MIGRAÇÃO. Anais... ENSM, 1997, p. 291-300.

BENNETT, N. G.; HORIUCHI, S. Estimating the completeness of death registration in closed population. Population Index, n. 47, p. 207-221, 1981.

BERCOVICH, A.; MADEIRA, F. Descontinuidades demográficas, onda jovem e mercado de trabalho: uma comparação entre Brasil e Argentina. In: CONGRESSO DA ASSOCIAÇÃO LATINO AMERICANA DE POPULAÇÃO, 1. Anais... Abep, 2004.

CAI, Y. An assesment of China's fertility level using the variable variable-r method. Demography, v. 45, n. 3, p. 271-281, May 2008.

CAMARANO, A. A. Envelhecimento da população brasileira: uma contribuição demográfica. Brasília: Instituto de Pesquisa Econômica Aplicada - Ipea, jan. 2002 (Texto para discussão nº 858).

CARVALHO, J. A. M. O saldo dos fluxos migratórios internacionais no Brasil na década de 80: uma tentativa de estimação. In: PATARRA, N. L. (Coord.). Migrações internacionais: herança XX, agenda XXI. Campinas: FNUAP (Programa interinstitucional de avaliação e acompanhamento das migrações internacionais; v. 2), 1996, p. 227-238.

Crescimento populacional e estrutura demográfica no Brasil. Belo Horizonte: Centro de Desenvolvimento e Planejamento Regional - Cedeplar/UFMG, 2004 (Texto para discussão n 227).

CARVALHO, J. A. M.; GARCIA, R. A. O envelhecimento da população brasileira: um enfoque demográfico. Cadernos de Saúde Pública, v. 19, n. 3, p. 725-733, 2003.

CARVALHO, J. A. M.; WONG, L. L. R. A transição da estrutura etária da população brasileira na primeira metade do século XXI. Cadernos de Saúde Pública, v. 24, n. 3, p. 587-605, 2008.

CARVALHO, J. A. M.; CAMPOS, M. B. O saldo migratório internacional do Brasil na década de 1990. In: ENCONTRO NACIONAL SOBRE MIGRAÇÃO, 5. Anais... São Paulo: Abep, 2007.

CELADE. Proyecciones de población a largo plazo. Observatório Demográfico, Ano VI, n. 11, 2011.

COALE, A. J.; CASELLI, G. Estimation of the number of persons at advanced ages from the number of deaths at each age in the given year and adjacent years. Genus, v. LXVI, n. 1/2,1990.

DIAS JUNIOR, C. S.; COSTA, C. S. O envelhecimento da população brasileira: uma análise de conteúdo das páginas da REBEP. In: ENCONTRO NACIONAL DE ESTUDOS POPULACIONAIS, 15. Anais... Abep, 2006.

FÍGOLI, M. G. B.; WONG, L. L. R. El camino hacia la estabilización demográfica y el proceso de envejecimiento en América Latina: una ilustración a partir de alguns países seleccionados. Papeles de Población, v. 1, n. 35, p. 32-46, 2003.

HORIUCHI, S.; PRESTON, S. H. Age-specific growth rates: the legacy of past population. Dynamics Demography, v. 25, n.3, p. 429-441, ago. 1988.

IBGE. Evolução e perspectivas da mortalidade infantil no Brasil. Rio de Janeiro: IBGE/Departamento de População e Indicadores Sociais, 1999 (Série Estudos e Pesquisas. Informação Demográfica e Socioeconômica, n. 2). 
JAVIQUE, D. C. R.; TURRA, C. M.; BONIFÁCIO, G. M. O.; WAJNMAN, S. Efectos de la migración sobre el crescimiento poblacional a largo plazo de las provincias cubanas. Revista Brasileira de Estudos de População, v. 30, 2, 2013.

MERLI, G. Mortality in Vietnam, 1979-1989. Demography, v. 35, n. 3, p. 345-60, 1988.

MOREIRA, M. M. Envelhecimento da população brasileira. Tese (Doutorado em Demografia) - Centro de Desenvolvimento e Planejamento Regional, Universidade Federal de Minas Gerais, Belo Horizonte, 1997.

MOREIRA, M. M.; CARVALHO, J. A. M. Envelhecimento da população e aposentadoria por idade. Previdência em Dados, v. 7, n. 4, p. 27-39, 1992.

MUNIZ, J. O. Efeitos das descontinuidades demográficas sobre o mercado de trabalho dos jovens: uma análise temporal utilizando-se painéis metropolitanos agrupados. Dissertação (Mestrado em Demografia) - Centro de Desenvolvimento e Planejamento Regional, Universidade Federal de Minas Gerais, Belo Horizonte, 2002.

MYRRHA, L. J. D. Estrutura etária brasileira: decomposição segundo variações na fecundidade e na mortalidade. Dissertação (Mestrado em Demografia) - Centro de Desenvolvimento e Planejamento Regional, Universidade Federal de Minas Gerais, Belo Horizonte, 2009.

PRESTON, S. H.; WANG, H. Intrisic growth rates and net reproduction rates in the presence of migration. Population and Development Review, v. 33, n. 4, December 2007.

PRESTON, S. H.; COALE, A. J. Age structure, growth, attrition and accession: a new synthesis. Population Index, v. 48, n. 2, p. 217-59, 1982.

PRESTON, S. H.; HEUVELINE, P.; GUILLOT, M. Demography: meansuring and modeling population processes. 1ed. Blackwell Publishing Ltd, 2001, p. 291.

PRESTON, S. H. Relations among standard epidemiologic measures in a population. American Journal of Epidemiology, v. 126, n. 2, p. 336-45, 1987.

WONG, L. L. R.; CARVALHO, J. A. M. O rápido processo de envelhecimento populacional no Brasil: sérios desafios para as políticas públicas. Revista Brasileira de Estudos de População, v. 23, n. 1, p. 5-26, jan./jun. 2006.

\section{Sobre os autores}

Luana Junqueira Dias Myrrha é doutora em Demografia pelo Centro de Desenvolvimento e Planejamento Regional - Cedeplar/UFMG e professora adjunta da Universidade Federal do Rio Grande do Norte.

Pamila Cristina Lima Siviero é doutora em Demografia pelo Centro de Desenvolvimento e Planejamento Regional - Cedeplar/UFMG e professora adjunta da Universidade Federal de Alfenas.

Simone Wajnman é doutora em Demografia pelo Centro de Desenvolvimento e Planejamento Regional - Cedeplar/UFMG e professora do Departamento de Demografia/Cedeplar, da Universidade Federal de Minas Gerais - UFMG.

Cássio M. Turra é doutor em Demografia pela Universidade da Pensilvânia e professor adjunto do Departamento de Demografia/Cedeplar, da Universidade Federal de Minas Gerais - UFMG.

\section{Endereço para correspondência:}

Cedeplar-FACE-UFMG

Av. Antônio Carlos, 6627

31270-901 - Belo Horizonte- MG, Brasil 


\begin{abstract}
The use of growth rates by age to identify the main stages of the demographic transition in Brazil

The analysis of the set of age specific growth rates in a population, proposed by Horiuchi and Preston (1988), is an alternative way to examine the process of changing age structure as well as to examine potential "marks" left by the demographic history of each cohort. The objective of this study is to apply this methodology to the Brazilian population and discuss some of the major demographic changes on the basis of growth rates seen in Brazil since the 1970s. Based on patterns of specific growth rates simulated by these authors to describe the structure of specific growth rates for each type of demographic changes, we evaluate possible stages in the country's demographic history that may be present in the current cohorts. The results show that the information contained in specific growth rates are quite instructive regarding the effects of variations in demographic regimes and is very useful for populations where vital rates are unreliable or unavailable.
\end{abstract}

Keywords: Age specific growth rates. Population aging. Mortality. Fertility.

\title{
Resumen
}

El uso de las tasas de crecimiento por edad para la identificación de las principales etapas de la transición demográfica en Brasil

El análisis del conjunto de las tasas específicas de crecimiento por edad de una población, propuesto por Horiuchi y Preston (1988), es una forma alternativa de investigar el proceso de cambio de la estructura etaria de una población y de examinar las posibles "marcas" que ha dejado la historia demográfica de cada cohorte. El objetivo de este trabajo es aplicar esta metodología a la población brasileña, analizando los grandes cambios demográficos observados en el país desde 1970 hasta la actualidad sobre la base de las tasas de crecimiento poblacionales. A partir de los patrones de las funciones de las tasas específicas de crecimiento, simuladas por esos autores para describir la estructura de estas tasas para cada régimen de cambio demográfico, se evaluaron las posibles marcas que la historia demográfica ha dejado en las actuales cohortes brasileñas. Los resultados demuestran que la información contenida en las tasas específicas de crecimiento es bastante esclarecedora de los efectos de las variaciones en los regímenes demográficos, y es muy útil en poblaciones cuyas tasas vitales no son confiables o no están disponibles.

Palabras clave: Tasas específicas de crecimiento. Envejecimiento poblacional. Mortalidad. Fecundidad.

Recebido para publicação em 05/09/2012

Aceito para publicação em 12/09/2014 
